\title{
Multiprofessional Medication Management (MuMM) Intervention Aiming at Enhancing the Tolerability of Cancer Chemotherapy: A Randomized Feasibility Trial
}

\author{
André Wilmer \\ University of Bonn \\ Corinna Vucur \\ University of Bonn \\ Andrea Tasar \\ Kronen Pharmacy Marxen Wesseling \\ Katharina Klima \\ Kronen Pharmacy Marxen Wesseling \\ Katrin Fleckenstein \\ Johanniter-Krankenhaus Bonn \\ Marie-Therese Puth \\ Universitatsklinikum Bonn
}

\section{Sandra Mitchell}

National Cancer Institute

Klaus Ruberg

Kronen Pharmacy Marxen Wesseling

Matthias Schmid

Universitatsklinikum Bonn

Yon D. Ko

Johanniter-Krankenhaus Bonn

Ulrich Jaehde ( $\nabla$ u.jaehde@uni-bonn.de)

University of Bonn https://orcid.org/0000-0002-2493-7370

\section{Research article}

Keywords: Adverse drug events, cancer care, medication review, medication management, symptoms

Posted Date: September 18th, 2020

DOI: https://doi.org/10.21203/rs.3.rs-37450/v2 
License: (c) (i) This work is licensed under a Creative Commons Attribution 4.0 International License. Read Full License 


\section{Abstract}

Background: A newly developed multiprofessional medication management (MuMM) intervention in an oncologic outpatient care setting was evaluated for its feasibility to enhance the tolerability of cancerdirected therapy. The intervention consisted of a basic care module for medication review including drugdrug interaction checks, and four supplementary modules for the management of common adverse events, specifically nausea/vomiting, mucositis, fatigue, and pain.

Methods: A single-centre, unblinded, randomized two-arm trial was conducted in the oncologic outpatient ward of a municipal hospital in Bonn, Germany, with an associated community pharmacy. Feasibility of the intervention was assessed by analyzing the application of supplementary modules and semistructured interviews with the participating health care providers. Symptom burden was assessed as primary outcome by the Patient-Reported Outcomes version of the Common Terminology Criteria for Adverse Events (PRO-CTCAE). Furthermore, health-related quality of life and patient satisfaction with received information were analyzed.

Results: 51 patients were randomized either to a control group receiving standard care ( 25 patients) or the intervention group receiving MuMM (26 patients). The results indicate that the MuMM intervention delivered by physicians, pharmacists, and nurses is needed and feasible, but hampered by several barriers concerning cooperation and communication within the multiprofessional health care team. The intervention did not affect symptom burden, quality of life, and patient satisfaction.

Conclusion: The degree of implementation of the MuMM intervention was apparently not high enough to show a preliminary efficacy signal. Nevertheless, our study provides a starting point and methodological framework for future trials aiming at enhancing the tolerability of cancer-directed therapy.

\section{TRIAL REGISTRATION NUMBER}

DRKS00010128 at the German Clinical Trials Register (DRKS)

Date of registration: March $7^{\text {th }}, 2016$ (retrospectively registered)

\section{Background}

Cancer patients undergoing chemotherapy are at high risk for drug-related toxicity and further drugrelated problems because of the complexity of chemotherapy regimens, typically including several different anticancer agents, each with its own toxicity profile. Additionally, supportive therapy is required to avoid or attenuate therapy-associated toxicity, which makes the treatment regimen even more complex. Further complicating the problem is the older age of many cancer patients. Elderly patients often have comorbid conditions, and the management of these conditions often introduces polymedication [1]. Cancer patients have been shown to take a median of five to nine prescribed drugs before starting 
anticancer treatment [2]. Furthermore, complementary and integrative therapy use is also common among older cancer patients [3].

As a consequence, cancer patients are exposed to a high risk for drug-related problems like drug-drug and drug-complementary and integrative therapy (CIT) interactions, and adverse drug reactions. These drugrelated problems are of high relevance for patient safety and can lead to unnecessary toxicity [4]. Moreover, elderly cancer patients have age-related physiological and functional changes like decreased renal and hepatic function. This may further increase the risk for drug-related toxicity and compromise treatment success $[2,5]$.

To enhance tolerability and limit the adverse effects of cancer treatment and drug-related problems, various strategies are required, including stronger patient involvement in symptom monitoring and better education and counseling of the patient regarding drug treatment [6-8]. Other effective measures are the participation of pharmacists on ward rounds, the integration of pharmacists into a medication management, and the performance of a thorough medication history for every patient $[9,10]$. Besides, the change from individual and independent care to collaborative multiprofessional teamwork is essential to identify and minimize risks within the whole medication process [11, 12]. The establishment of multiprofessional teams increases the continuity of the care process as well as the coordination and communication across the health care professions and is demanded in cancer care by international medical societies [13-15]. Promising results on multiprofessional cancer care including a pharmacist could be obtained in a previous study of our group, showing improved response to the antiemetic prophylaxis in breast or ovarian cancer patients [16]. To further enhance collaboration, we decided to extend it to the nurse profession and to implement the multiprofessional approach from the beginning, i.e. already at the point of development of the intervention.

The aim of this project was to develop a new multiprofessional medication management intervention and to evaluate its feasibility and potential to reduce severe chemotherapy-associated symptom burden. Several distinct strategies including facilitating interprofessional cooperation, optimizing the supportive therapy, and strengthening the patient role were combined to a new care intervention and implemented in an oncologic outpatient clinic of a German municipal hospital with pharmaceutical care services provided by a community pharmacy.

Our study adheres to the CONSORT guidelines. A completed CONSORT checklist is provided as Suppl. 1.

\section{Methods}

\section{Study design and setting}

This trial is a single-centre, unblinded, randomized two-arm study with a nested qualitative study. A multiprofessional medication management (MuMM) intervention was implemented at an oncologic outpatient ward of the Johanniter-Hospital, a municipal hospital in Bonn, Germany, to assess its 
feasibility in clinical practice. The hospital does not have an own pharmacy, pharmaceutical services are provided by a community pharmacy.

The recruitment period was from November 2012 until January 2014. Patients were enrolled by the study team after signing written informed consent and the randomization was carried out in terms of a computer-generated block randomization with variable block size by a staff member not involved in the study. Because of the nature of the intervention, including repeated medication reviews and counseling, patients and medical staff could not be blinded. Data collection and assessment were not blinded either. Patients were observed for a maximum of five chemotherapy cycles. The sample size was set to 50 patients aiming at evaluating the feasibility of a full-scale efficacy study.

\section{Participants}

Eligible patients were randomized 1:1 either to the control group receiving standard care, or to the intervention group receiving the MuMM intervention. Inclusion criteria required that patients were age 18 or older, had been diagnosed with a solid tumor, were receiving anticancer drug therapy, and were able to speak, read and write German. Exclusion criteria included a diagnosis of a hematologic malignancy, the presence of comorbid conditions (e.g. Alzheimer's disease) that would preclude the ability to provide informed consent or limit comprehension of the patient-reported outcome measures and the intention to change the location of treatment in the near future.

\section{Intervention development}

To develop a structured and standardized medication management for a multiprofessional team of physicians, nurses, and pharmacists, the current evidence on medication management and supportive care was searched by a detailed literature review. The medication management process was based on a modular approach. Hence, different care modules were developed including a basic care module and four supplementary modules. The basic care module corresponds to the core components of a medication management. These are a comprehensive medication review including an interaction check and the identification of drug-related problems, patient counseling on their medication, and the provision of a written medication plan. A prescreening for chemotherapy-induced toxicity is also part of the basic care module. The four supplementary modules comprise the management of the common adverse drug reactions nausea/vomiting, mucositis, fatigue, and pain. Each supplementary module consists of a bundle of measures on three different levels of the medication process: (1) the therapy, (2) the patient, and (3) the health care providers. The therapy level is addressed by written evidence-based recommendations for supportive therapy of the corresponding toxicity. At the patient level written leaflets for patient information and counseling on prevention and therapy of the corresponding symptoms are to support the patient in taking a more active role. And finally, the level of the health care providers is addressed by care algorithms illustrating every step of the care process and defining and allocating tasks and responsibilities to the different members of the multiprofessional care team (see Figure 1 for the structure of the MuMM intervention). The individual modules were discussed and finalized in several meetings consisting of at least one physician, one pharmacist, and one nurse. 


\section{Study intervention}

Patients in the control arm received standard care according to the current practice in Germany where pharmacists usually (and especially in the study centre) do not act at the bedside and do not have direct contact to patients on the ward. Moreover, structured exchange and communication between physicians, pharmacists and nurses regarding medication safety are not common so far.

Participants randomized to the intervention arm received the modular MuMM intervention. After the randomization to the intervention group the basic care module was applied to every patient by the study pharmacist. Within a patient interview, a comprehensive medication reconciliation was conducted and the patient was informed and counseled in detail regarding his or her therapy and possible adverse drug reactions. Subsequent to the patient interview, a medication review focusing on drug-drug interactions was conducted, and a written medication plan was compiled. In case of identified medication-related interactions, the medication was adjusted in collaboration with the responsible physician. The basic module was applied by the study pharmacist after patient inclusion and at the beginning of every new cycle until the fifth cycle of chemotherapy. In the case that patients showed symptoms like nausea/vomiting, mucositis, fatigue or pain, the corresponding supplementary modules were initiated by the pharmacist (see Figure 2 for the course of the intervention). The modules for nausea/vomiting and mucositis were also initiated if the chemotherapy had a low to high emetogenic risk or the patient had a risk $>10 \%$ to develop severe mucositis, respectively. All supplementary modules included the evidencebased supportive therapy for the corresponding adverse drug reaction in close cooperation between all health care professions as well as patient counseling with regard to non-pharmacological and pharmacological self-care measures to prevent or alleviate symptoms. Patient counseling was supported by the provision of written information and documentation material in terms of information booklets. The algorithms of the supplementary modules are provided as supplementary information (Suppl. 2-5).

\section{Feasibility assessment}

The feasibility of the intervention by the different health care providers was assessed by analyzing the application of the different care modules, i.e. the frequency of patient counseling within the basic care module, the extent of delivery of written medication plans, the number of patients with active modules, and the number of applied modules per patient.

Moreover, the experience gained by the clinical staff after implementation of the MuMM intervention was evaluated by means of semi-structured interviews with open questions. Hence, questions were prespecified but changes in sequence and formulation as well as further enquiry were allowed. The interviews were recorded, transcribed, and the main statements of the participants categorized and coded by intuitive coding according to a qualitative content analysis with the Software MAXQDA 11 (VERBI $\mathrm{GmbH}$, Berlin).

\section{Outcome measures}




\section{Symptom burden}

Symptom burden was defined as primary outcome and assessed according to the Patient-Reported Outcomes version of the Common Terminology Criteria for Adverse Events (PRO-CTCAE ${ }^{T M}$ ) of the National Cancer Institute (NCI), USA [17]. The PRO-CTCAE item library includes items that capture the full range of symptomatic treatment effects that may be experienced across a variety of disease sites and cancer treatment modalities $[18,19]$. It was validated in a large population of cancer patients in the USA [20].

A set of 31 items drawn from the translated German PRO-CTCAE item library has been evaluated concerning its quantitative measurement properties [21]. Out of the validated German PRO-CTCAE item set 19 items were selected for this study. 11 out of these 19 items focusing on the five anticancer treatment-related symptoms nausea, vomiting, mucositis, fatigue, and pain, were further analyzed as they were directly targeted by the four supplementary modules of the intervention. The recall period was the past seven days. Symptom burden was assessed after randomization (cycle 0 ) and at the beginning of every new cycle for a maximum of five cycles (cycle 1 to 5 ).

\section{Health-related quality of life and patient satisfaction with information}

Further secondary outcomes were health-related quality of life (HRQoL) and patient satisfaction with the received information. Health-related quality of life was assessed with the global health status scale of the QLQ-C30 V 3.0 questionnaire from the European Organization for Research and Treatment of Cancer (EORTC). The QLQ-C30 is a validated questionnaire particularly developed for cancer patients [22]. HRQoL of the patients was assessed after randomization and before first treatment (cycle 0 ) and at the beginning of every new treatment cycle retrospectively for the last 7 days for a maximum of five cycles (cycle 1 to 5).

The satisfaction of patients with the information about their disease and treatment was assessed with the validated German version of the Patient Satisfaction with Cancer Treatment Education Questionnaire (PS-CaTE) [23]. The questionnaire consists of four subscales assessing satisfaction with information on cancer treatment, adverse effects, complementary treatment options, and information sources. Overall satisfaction with cancer treatment education is computed as the mean of all subscales; the theoretical score range is 1 to 5 , with higher scores indicating greater satisfaction [23]. Patient satisfaction with information was assessed after randomization and at the end of cycle 5 or at the patient's last treatment cycle if he or she received less than five cycles of chemotherapy.

\section{Statistical analysis}

The original study protocol aimed at evaluating the influence of the MuMM intervention on the proportion of patients suffering from at least one severe symptomatic toxicity as primary outcome. A formal sample size calculation was performed resulting in a number of 74 patients based on an assumed reduction of the primary outcome by $50 \%$, a significance level of $5 \%$, a power of $80 \%$, and a drop-out rate of $20 \%$. 
However, the planned sample size could not be reached due to termination of the recruitment period. In order to allow the full exploitation of the information content of the data from the 51 available patients we decided to extend the analysis of the primary outcome and evaluated the time-dependence of symptom burden using generalized estimating equation (GEE) models for each symptom targeted by the intervention.

For this purpose, scores for PRO-CTCAE item clusters were calculated per cycle according to the following equations. The composite PRO-CTCAE item clusters are specified in Table 1. The raw score (RS) was formed by the average of the items $I_{1,2, \ldots, n}$ that contribute to a cluster:

Eq. 1

$$
R S=\frac{I_{1}+I_{2}+\ldots+I_{n}}{n}
$$

The scores for PRO-CTCAE item clusters were standardized by a linear transformation to a scale from 0 to 100 according to Equation 2 with higher scores indicating a higher symptom burden:

Eq. 2

$$
\text { Score }=\left\{\frac{R S}{\text { ronge }}\right\} \times 100,
$$

where range refers to the difference between the maximum possible value and the minimum possible value of the answer options. In cases of missing data, scores for PRO-CTCAE item clusters were calculated if at least $50 \%$ of the item values were available [21].

Data entry and statistical data analysis were carried out using Excel ${ }^{\mathrm{TM}} 2007$ (Microsoft, Redmond, Washington, USA), SPSS, version 22 (SPSS Inc., Chicago, Illinois, USA), SAS, version 9.3 (SAS Institute Inc., Cary, North Carolina, USA), and R, version 3.3.3 [24]. Descriptive statistics (means, standard deviations, medians, ranges, counts, percentages) was used to characterize the patient population and summarize the study results. The level of significance was set to $5 \%(p<0.05)$ for all analyses.

\begin{tabular}{|c|c|c|}
\hline Item cluster & Number of items & Item dimensions \\
\hline Nausea & 2 & Frequency $^{\mathrm{a}}$, severity ${ }^{\mathrm{b}}$ \\
\hline Vomiting & 2 & Frequency $^{\mathrm{a}}$, severity ${ }^{\mathrm{b}}$ \\
\hline Mucositis & 2 & Severity $^{b}$, interference ${ }^{c}$ \\
\hline Fatigue & 2 & Severity $^{b}$, interference ${ }^{c}$ \\
\hline Pain & 3 & Frequency $^{\mathrm{a}}$, severity ${ }^{\mathrm{b}}$, interference ${ }^{\mathrm{c}}$ \\
\hline
\end{tabular}

Table 1: PRO-CTCAE item clusters for symptom terms 
a Score range from 'never' (0) to 'almost constantly' (4); 'Score range from 'none' (0) to 'very severe' (4); 'Score range from 'not at all' (0) to 'very much' (4)

Repeated measures of symptom scores (nausea, vomiting, mucositis, fatigue, pain) formed according to equations 1 and 2 were used as dependent variables in the GEE models. Covariates included in the analysis were treatment group, therapy cycle, and treatment modification (yes/no, defined by dose reductions, dose delays, adjustments of therapy, or discontinuation of therapy). Generalized score tests were used to analyze the effects of covariates on symptom scores. For each symptom score a separate model was fitted.

\section{Results}

\section{Participant recruitment and characteristics}

A total of 96 patients were assessed for eligibility for this study during the data collection period. Fiftyone patients were enrolled in the study. The patient recruitment process is shown in Figure 3.

Twenty-six patients were analyzed in the intervention group and 25 in the control group. Table 2 shows the socio-demographic and disease-related patient characteristics. The two study groups were well balanced.

Table 2: Socio-demographic and disease-related patient characteristics 


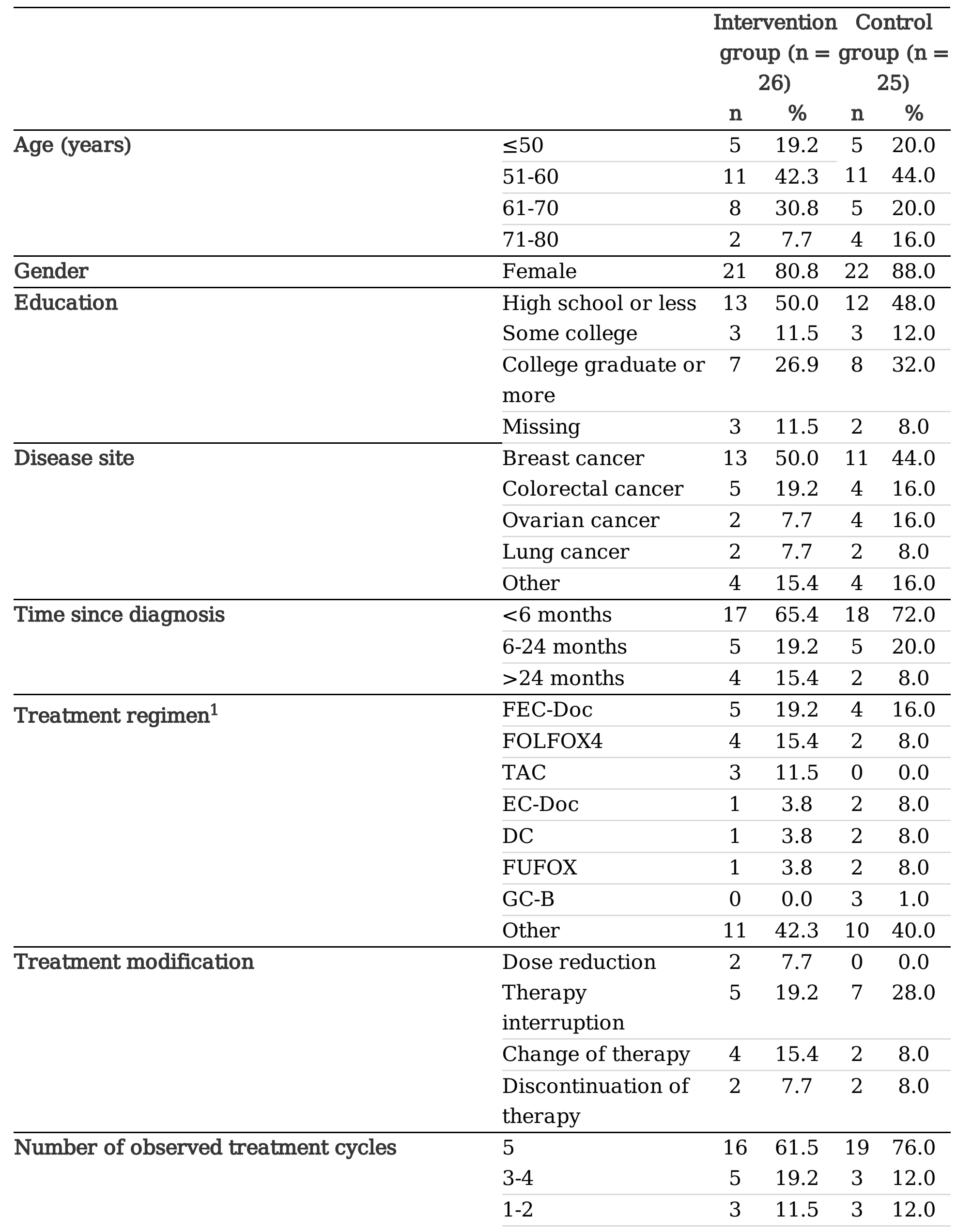


Number of simultaneously taken / administered 6-10

2

$\begin{array}{lll}7.7 & 0 & 0.0\end{array}$

\begin{tabular}{lccccc} 
drugs in total & $11-15$ & 13 & 50.0 & 12 & 48.0 \\
\cline { 2 - 6 } & $>15$ & 3 & 11.5 & 2 & 8.0 \\
\hline
\end{tabular}

1 Therapy regime: FEC-Doc $=$ Fluorouracil + Epirubicin + Cyclophosphamide followed by Docetaxel; FOLFOX4 = Calcium folinate + Fluorouracil + Oxaliplatin $;$ TAC $=$ Docetaxel + Doxorubicin + Cyclophosphamide EC-Doc $=$ Epirubicin + Cyclophosphamide followed by Docetaxel; $D C=$ Docetaxel + Cyclophosphamide FUFOX $=$ Fluorouracil + Calcium folinate + Oxaliplatin; $G C-B$ = Carboplatin + Gemcitabin + Bevacizumab

\section{Module application}

Twenty-six patients were randomized to get the MuMM intervention. These patients were counseled in total 66 times by the pharmacist. The mean duration of the patient interviews was 16 minutes with a range of 5 to 50 minutes. Every patient received a written medication plan.

The supplementary modules for mucositis, fatigue, nausea/vomiting, and pain were applied in 18, 18, 16, and 11 patients, respectively. Most patients had three active supplementary modules and four patients had no active supplementary module.

\section{Semi-structured interviews}

The interviews were conducted with the core team consisting of a physician, a nurse, and a pharmacist who were primarily providing the multiprofessional medication management. In general, all three were satisfied with the preparation process. All of them used the written leaflets for patient information and counseling. The physician appreciated the pharmacist very much for the comprehensive medication review and identification of drug-related problems. All involved professionals emphasized that the patient interviews during the intervention intensified the involvement of the patients. However, the transfer of the intervention to clinical practice revealed some problems and barriers interfering with the optimal application of the multiprofessional medication management. From the physician's point of view there was often not enough time for proper patient interviews and documentation regarding symptoms and adverse drug reactions as demanded for the medication management and as requirement for the supplementary modules of the intervention. The nurse felt herself only poorly integrated into the multiprofessional health care team and in some occasions did not know if she should contact the physician or the pharmacist. The pharmacist perceived the communication with the physician as limited and as mainly taking place within the written patient records. She missed a feedback from the physician concerning her documentation of the medication management in the patient records.

All three interviewed health care providers suggested changes for improvement of the MuMM intervention. These were: 
- to use a digital hub for central documentation of patient data with access from the outpatient ward as well as from the pharmacy to optimize and facilitate communication,

- to implement patient consultation via telephone by the pharmacist or a regular consultation-hour by the pharmacist for patients at the outpatient ward once a week,

- to include oral anticancer therapies and further symptoms like neuropathic symptoms into the MuMM intervention, and

- to implement meetings with all participating health care providers on a regular basis to discuss specific patient cases and to discuss the further care process for the individual patient.

\section{Symptom burden}

Figure 4 shows the proportion of patients per cycle and treatment group suffering from nausea, vomiting, mucositis, fatigue, or pain of PRO-CTCAE scores $\geq 75$, corresponding to grade 3 or 4 (severe or very severe; frequently or almost constantly; quite a bit or very much) symptomatic toxicity. There was a relatively high symptom burden at baseline for fatigue and pain; $20 \%$ of the patients in the control group and $8 \%$ of the patients in the intervention group reported PRO-CTCAE scores $\geq 75$ for both symptoms at cycle 0 . In total, a median of seven patients (34\%, range 5-9) in the intervention group and of eight patients (43\%, range 6-14) in the control group suffered from toxicity of PRO-CTCAE score $\geq 75$ nausea, vomiting, mucositis, fatigue, or pain within the five cycles of chemotherapy.

To analyze the effect of the MuMM intervention on symptom burden we used GEE models. Table 3 shows the parameter estimates and the $p$ values obtained from the GEE models to analyze the associations between the defined predictor variables and the occurrence of the symptoms nausea, vomiting, mucositis, fatigue, or pain. No statistically significant associations could be found for any of the symptoms with the variables treatment group, therapy cycle, or treatment modification except for the association between nausea and the therapy cycle ( 1 to 3 ) as well as mucositis and the therapy cycle ( 1 to 5 ).

Table 3: Association between predictor variables and the outcomes nausea, vomiting, mucositis, fatigue and pain: parameter estimates ( $($ ), standard error (SE), 95\% confidence interval (CI) and p values based on GEE analyses 


\begin{tabular}{|c|c|c|c|c|c|c|c|c|}
\hline \multirow[t]{2}{*}{ Predictor variable } & \multicolumn{4}{|c|}{ Nausea } & \multicolumn{4}{|c|}{ Vomiting } \\
\hline & $ß$ & $\mathrm{SE}$ & $95 \% \mathrm{CI}$ & $\begin{array}{c}\mathrm{P} \\
\text { value }\end{array}$ & $\S$ & $\mathrm{SE}$ & $95 \% \mathrm{CI}$ & $\begin{array}{c}\mathrm{P} \\
\text { value }\end{array}$ \\
\hline Intercept & 11.10 & 3.44 & $4.34-17.85$ & 0.001 & 3.24 & 2.17 & $-1.01-7.50$ & 0.136 \\
\hline Intervention group ${ }^{\mathrm{a}}$ & -5.47 & 4.93 & $-15.13-4.19$ & 0.267 & 0.15 & 1.89 & $-3.55-3.86$ & 0.936 \\
\hline Cycle $1^{b}$ & 9.47 & 3.81 & $2.01-16.93$ & 0.013 & 2.23 & 2.63 & $-2.92-7.37$ & 0.397 \\
\hline Cycle $2^{b}$ & 12.89 & 4.42 & $4.22-21.57$ & 0.004 & -1.17 & 2.24 & $-5.56-3.22$ & 0.600 \\
\hline Cycle $3^{b}$ & 11.88 & 4.63 & $2.81-20.96$ & 0.010 & 0.39 & 3.22 & $-5.92-6.70$ & 0.904 \\
\hline Cycle $4^{\mathrm{b}}$ & 5.66 & 4.11 & $-2.39-13.71$ & 0.168 & -3.04 & 1.70 & $-6.37-0.30$ & 0.074 \\
\hline Cycle $5^{b}$ & 6.82 & 4.18 & $-1.36-15.01$ & 0.102 & -3.02 & 1.70 & $-6.35-0.31$ & 0.075 \\
\hline Treatment modification ${ }^{\mathrm{c}}$ & 0.55 & 4.73 & $-8.71-9.82$ & 0.907 & -1.71 & 0.88 & $-3.44-0.02$ & 0.053 \\
\hline
\end{tabular}

\begin{tabular}{|c|c|c|c|c|c|c|c|c|}
\hline \multirow[t]{2}{*}{ Predictor variable } & \multicolumn{4}{|c|}{ Mucositis } & \multicolumn{4}{|c|}{ Fatigue } \\
\hline & $\S$ & SE & $95 \%$ CI & $\begin{array}{c}\mathrm{P} \\
\text { value }\end{array}$ & $\S$ & SE & $95 \% \mathrm{CI}$ & $\begin{array}{c}\mathrm{P} \\
\text { value }\end{array}$ \\
\hline Intercept & 3.03 & 2.62 & $-2.11-8.17$ & 0.248 & 29.70 & 5.18 & $19.53-39.83$ & $<0.001$ \\
\hline Intervention group ${ }^{\mathrm{a}}$ & -3.06 & 3.78 & $-10.47-4.36$ & 0.419 & 2.42 & 5.71 & $-8.77-13.62$ & 0.671 \\
\hline Cycle $1^{\mathrm{b}}$ & 15.30 & 3.96 & $7.53-23.07$ & $<0.001$ & 5.12 & 3.45 & $-1.66-11.87$ & 0.139 \\
\hline Cycle $2^{b}$ & 11.27 & 2.98 & $5.43-17.12$ & $<0.001$ & 7.20 & 3.92 & $-0.50-14.89$ & 0.067 \\
\hline Cycle $3^{b}$ & 13.41 & 4.03 & $5.51-21.31$ & 0.001 & 7.37 & 5.38 & $-3.17-17.90$ & 0.171 \\
\hline Cycle $4^{b}$ & 10.57 & 3.47 & $3.77-17.37$ & 0.002 & 6.34 & 4.55 & $-2.57-15.25$ & 0.163 \\
\hline Cycle $5^{b}$ & 10.94 & 3.59 & $3.90-17.98$ & 0.002 & 8.11 & 5.38 & $-2.43-18.65$ & 0.132 \\
\hline Treatment modification $^{\mathrm{c}}$ & -6.13 & 4.22 & $-14.42-2.14$ & 0.146 & 12.28 & 6.62 & $-0.71-25.26$ & 0.064 \\
\hline
\end{tabular}




\begin{tabular}{lcccc}
\hline Predictor variable & \multicolumn{4}{c}{ Pain } \\
& $\Re$ & SE & $95 \%$ CI & $\begin{array}{c}\text { P } \\
\text { value }\end{array}$ \\
\hline Intercept & 30.13 & 4.76 & $20.80-39.47$ & $<0.001$ \\
\hline Intervention group $^{\mathrm{a}}$ & 2.19 & 5.69 & $-8.95-13.34$ & 0.700 \\
\hline Cycle 1 $^{\mathrm{b}}$ & & & & \\
\hline Cycle 2 $^{\mathrm{b}}$ & -2.45 & 4.00 & $-10.29-5.39$ & 0.540 \\
\hline Cycle 3 $^{\mathrm{b}}$ & -3.07 & 4.69 & $-12.26-6.11$ & 0.512 \\
\hline Cycle 4 $^{\mathrm{b}}$ & & & & \\
\hline Cycle 5 $^{\mathrm{b}}$ & -1.77 & 5.48 & $-12.50-8.97$ & 0.747 \\
\hline Treatment modification $^{\mathrm{c}}$ & -6.58 & 5.57 & $-17.50-4.35$ & 0.238 \\
\hline & -0.31 & 5.27 & $-10.63-10.01$ & 0.953 \\
\hline
\end{tabular}

${ }^{a}$ Reference group: control group; ${ }^{b}$ Reference group: Cycle $0 ;{ }^{c}$ Reference group: no Note: All GEE models included treatment group, therapy cycle, and treatment modification.

Non-significant reductions of symptom burden in the intervention group were estimated for nausea and mucositis. Treatment modification, i.e. dose reduction, therapy interruption, change of therapy, or discontinuation of therapy, led to lower estimates of symptom burden for vomiting, mucositis, and pain. There might have been interaction effects between therapy cycle and treatment modification, which however, could not be analyzed in our GEE models due to the relatively small sample size.

\section{Health-related quality of life and patient satisfaction with information}

Health-related quality of life is shown per cycle and group in Suppl. 6. We observed a large variability of health-related quality of life among patients. However, the differences between the two groups were small and not significant at any treatment cycle.

Overall satisfaction with information was already high at baseline with a mean score of 4.2 of 5.0 in the intervention group and 4.1 in the control group. This score could not be improved from cycle 0 to 5 within the two groups, with a mean difference of -0.2 ( $95 \% \mathrm{Cl}$ : -0.4 to 0.1$)$ in the intervention group $(n=16)$ and -0.1 (95\% Cl: -0.5 to 0.1$)$ in the control group $(n=18)$. No statistically significant difference was found between the two groups, neither for the total score nor for the four subscales. 


\section{Discussion}

In this study, we tested for the first time a newly developed MuMM intervention for standardized and structured medication management in outpatient cancer care. Due to the modular approach the intervention is very flexible and can be applied in a patient-tailored manner according to the individual medication, symptom status, and risk for toxicity. The study was conducted in terms of health services research with broad inclusion and minimal exclusion criteria allowing the formation of a heterogeneous patient sample.

The originally calculated sample size was not reached which is a significant limitation of the study. Nevertheless, the study design enabled the evaluation of the intervention in daily routine of an outpatient setting reflecting real-life conditions. The high rate of application of the individual care modules indicates that a MuMM intervention delivered by a team comprised of a physician, a nurse, and a pharmacist is needed and feasible. The use of GEE models to analyze the association between independent variables and the dependent outcome parameters permits to assess the influence of different factors on the outcome of interest in such a study setting and could be a framework for analysis in similar studies in the future.

As the results show, no significant improvements of symptom burden could be reached in cancer patients undergoing chemotherapy by means of the MuMM intervention. However, it cannot be concluded that the intervention is not effective. First, the study cohort was relatively heterogeneous as most adult patients with a solid tumor were eligible irrespective of their tumor entity and stage. Second, the number of parameters contained in the GEE models $(n=8)$ was rather large relative to the sample size $(n=51)$, so that the size of the study may have been too small to detect intervention effects at the $5 \%$ significance level. On the other hand, many of the GEE estimates were highly plausible in terms of direction and magnitude, implying that these estimates could be used in a next step to calculate the sample size of a future trial achieving higher power by larger sample sizes.

The interviews with the involved health care providers revealed several problems concerning the implementation of the intervention in clinical practice. The basic principles for effective multiprofessional patient care are clear responsibilities and accountabilities, shared goals, and effective communication [16]. Subsequent evaluations should therefore be focused on optimized preparation and training of the participating health care providers and intensify the support during implementation. Multiprofessional cooperation and communication certainly have to be intensified and well-rehearsed before initiating further efficacy evaluations of the presented intervention.

An important limitation associated with the single center study design is the risk of contamination between the groups. This could not be avoided completely since a strict spatial separation of the two study groups was not possible. Hence, information regarding prevention and treatment of symptoms could have been interchanged between patients of the intervention and control group. To avoid this risk and to provide significant findings, further trials to evaluate the effect of the multiprofessional care intervention should be designed multi-centered and cluster-randomized [25]. 
An improvement in health-related quality of life in the intervention arm compared to the control arm was not observed either. The score of the EORTC QLQ-C30 global health status scale decreased in both groups from cycle 0 to 5 . Nevertheless, there was a high variability in quality of life in both groups during the five study cycles, which is probably due to the heterogeneity of the patient cohort in the present study. Moreover, no difference could be found between the two groups for patient satisfaction with the information after the fifth study cycle. The overall satisfaction score was already high in both groups at baseline, leaving little score range to demonstrate improvement. It remained on that high level until cycle 5 and was in accordance with scores achieved in a previous study [16].

The MuMM intervention is based on a structured collaboration among physicians, nurses, and pharmacists. Diagnosis, treatment decision and adjustment are the main responsibility of the physician. The responsibilities of the nurse are routine patient care and to pass information concerning symptoms and drug-related problems from the patient to the physician and pharmacist. The pharmacist is primarily responsible for the comprehensive medication review and for provision of information and counseling of the patients. This task assignment was supported by a nationwide study in Germany about task allocation in multiprofessional cancer medication management [26]. The positive effect of integrating a pharmacist into a multiprofessional cancer care team for medication management has been shown already in several studies for in- and outpatient care [27, 28]. In subsequent projects inclusion of nutritionists, psycho-oncologists, and physical therapists should be considered to broaden the delivery of multiprofessional care. Moreover, additional modules could be integrated in the intervention.

\section{Conclusions}

A multiprofessional medication management intervention delivered by physicians, pharmacists, and nurses is feasible in a German municipal hospital with pharmaceutical care provided by a community pharmacy. The qualitative study provides information on barriers and optimization potential for a successful implementation of this complex intervention. The measured endpoints were not affected by the intervention suggesting that the degree of its implementation was not high enough to show a preliminary efficacy signal. Nevertheless, our trial provides a starting point and methodological framework for developing multiprofessional interventions aiming at enhancing the tolerability of cancerdirected therapy.

\section{Abbreviations}

CIT Complementary and integrative therapy

EORTC European Organization for Research and Treatment of Cancer

GEE Generalized estimating equation

HRQoL Health-related quality of life 
MuMM Multiprofessional Medication Management

PRO-CTCAE Patient-Reported Outcomes version of the Common Terminology Criteria for Adverse Events

QLQ-C30 Quality of Life Questionnaire-Core 30

RS Raw score

\section{Declarations}

Ethics approval and consent to participate: This study was performed in line with the principles of the Declaration of Helsinki. Approval was granted by the Ethics Committee at the Faculty of Medicine of the University of Bonn, Germany (project number 250/12). Informed consent was obtained from all individual participants included in the study.

Consent for publication: Not applicable.

Availability of data and materials: The dataset and the materials are available by emailing the corresponding author upon reasonable request.

Competing interests: The authors declare that they have no competing interests.

Funding: This research received no specific grant from any funding agency in the public, commercial or not-for-profit sectors.

Authors' contributions: AW, CV, and UJ designed the study. AW, AT, KK, KF, KR, and YDK were involved in data collection and provision of patient care. MTP, SAM, and MS contributed substantially to statistical data analysis and interpretation. The first draft of the manuscript was written by AW, CV, and UJ, and all authors commented on previous versions of the manuscript. All authors read and approved the final manuscript.

Acknowledgements: We thank all patients who participated in our study. Moreover, the excellent collaboration with all oncologists, pharmacists, and nurses is gratefully acknowledged.

\section{References}

1. Ko C, Chaudhry S (2002) The need for a multidisciplinary approach to cancer care. J Surg Res 105:53-57.

2. Lees J, Chan A (2011) Polypharmacy in elderly patients with cancer: clinical implications and management. Lancet Oncol 12:1249-1257.

3. Maggiore RJ, Gross CP, Hurria A (2010) Polypharmacy in older adults with cancer. Oncologist 15:507-522. 
4. Jaehde U, Liekweg A, Simons S, Westfeld M (2008) Minimising treatment-associated risks in systemic cancer therapy. Pharm World Sci 30: 161-168.

5. Blower P, Wit R de, Goodin S, Aapro M (2005) Drug-drug interactions in oncology: why are they important and can they be minimized? Crit Rev Oncol Hematol 55:117-142.

6. Jahn P, Renz P, Stukenkemper J, Book K, Kuss O, Jordan K et al. (2009) Reduction of chemotherapyinduced anorexia, nausea, and vomiting through a structured nursing intervention: a clusterrandomized multicenter trial. Support Care Cancer 17:1543-1552.

7. Riese C, Weiß B, Borges U Jr, Beylich A, Dengler R, Hermes-Moll K et al. (2017) Effectiveness of a standardized patient education program on therapy-related side effects and unplanned therapy interruptions in oral cancer therapy: a cluster-randomized controlled trial. Support Care Cancer 25:3475-3483.

8. Nipp RD, El-Jawahri A, Ruddy M, Fuh C, Temel B, D'Arpino SM et al. (2019) Pilot randomized trial of an electronic symptom monitoring intervention for hospitalized patients with cancer. Ann Oncol 30:274-280.

9. Nipp RD, Ruddy M, Fuh CX, Zangardi ML, Chio C, Kim EB et al. (2019) Pilot Randomized Trial of a Pharmacy Intervention for Older Adults with Cancer. Oncologist 24:211-218.

10. Dillmon MS, Kennedy EB, Anderson MK, Brodersen M, Cohen H, D Amato SL et al. (2020) PatientCentered Standards for Medically Integrated Dispensing: ASCO/NCODA Standards. J Clin Oncol 38:633-644.

11. Leape LL (2009) Errors in medicine. Clin Chim Acta 404:2-5.

12. Liekweg A, Westfeld M, Jaehde $U$ (2004) From oncology pharmacy to pharmaceutical care: new contributions to multidisciplinary cancer care. Support Care Cancer 12:73-79.

13. Fleissig A, Jenkins V, Catt S, Fallowfield L (2006) Multidisciplinary teams in cancer care: are they effective in the UK? Lancet Oncol 7:935-943.

14. Mitchell P, Wynia M, Golden R, McNellis B, Okun S, Webb CE et al. (2012) Core principles \& values of effective team-based health care. Institute of Medicine. https://nam.edu/wpcontent/uploads/2015/06/VSRT-Team-Based-Care-Principles-Values.pdf. Accessed 5 April 2020

15. ASCO-ESMO consensus statement on quality cancer care (2006) J Clin Oncol 24:3498-3499.

16. Liekweg A, Westfeld M, Braun M, Zivanovic O, Schink T, Kuhn W et al. (2012) Pharmaceutical care for patients with breast and ovarian cancer. Support Care Cancer 20:2669-2677.

17. National Cancer Institute (2020) Patient-Reported Outcomes version of the Common Terminology Criteria for Adverse Events (PRO-CTCAE ${ }^{\mathrm{TM}}$ ). https://healthcaredelivery.cancer.gov/pro-ctcae. Accessed 5 April 2020.

18. Basch E, Bennett A, Pietanza MC (2011) Use of patient-reported outcomes to improve the predictive accuracy of clinician-reported adverse events. J Natl Cancer Inst 103:1808-1810.

19. Hay JL, Atkinson TM, Reeve BB, Mitchell SA, Mendoza TR, Willis G et al. (2014) Cognitive interviewing of the US National Cancer Institute's Patient-Reported Outcomes version of the Common 
Terminology Criteria for Adverse Events (PRO-CTCAE). Qual Life Res 23:257-269.

20. Dueck AC, Mendoza TR, Mitchell SA, Reeve BB, Castro KM, Rogak LJ et al. (2015) Validity and Reliability of the US National Cancer Institute's Patient-Reported Outcomes Version of the Common Terminology Criteria for Adverse Events (PRO-CTCAE). JAMA Oncol 18: 1051-1059.

21. Hagelstein V, Ortland I, Wilmer A, Mitchell SA, Jaehde U (2016) Validation of the German patientreported outcomes version of the common terminology criteria for adverse events (PRO-CTCAE ${ }^{\mathrm{TM}}$ ). Ann Oncol 27: 2294-2299.

22. Aaronson NK, Ahmedzai S, Bergman B, Bullinger M, Cull A, Duez NJ et al. (1993) The European Organization for Research and Treatment of Cancer QLQ-C30: A quality-of-life instrument for use in international clinical trials in oncology. J Natl Cancer Inst 85:365-376.

23. Liekweg A, Eckhardt M, Taylor SC, Erdfelder E, Jaehde U (2005) Psychometric assessment and application of a questionnaire measuring patient satisfaction with information on cancer treatment. Pharm World Sci 27: 96-103.

24. R Core Team (2017) R: A language and environment for statistical computing. R Foundation for Statistical Computing, Vienna, Austria. https://www.R-project.org. Accessed 5 April 2020

25. Torgerson DJ (2001) Contamination in trials: is cluster randomisation the answer? BMJ 322:355-357.

26. Döhler N, Krolop L, Ringsdorf S, Meier K, Ko Y, Kuhn W et al. (2011) Task allocation in cancer medication management - integrating the pharmacist. Patient Educ Couns 83:367-374.

27. Bremberg ER, Hising C, Nylén U, Ehrsson H, Eksborg S (2006) An evaluation of pharmacist contribution to an oncology ward in a Swedish hospital. J Oncol Pharm Pract 12:75-81.

28. Simons S, Ringsdorf S, Braun M, Mey UJ, Schwindt PF, Ko YD et al. (2011) Enhancing adherence to capecitabine chemotherapy by means of multidisciplinary pharmaceutical care. Support Care Cancer 19: 1009-1018.

\section{Figures}




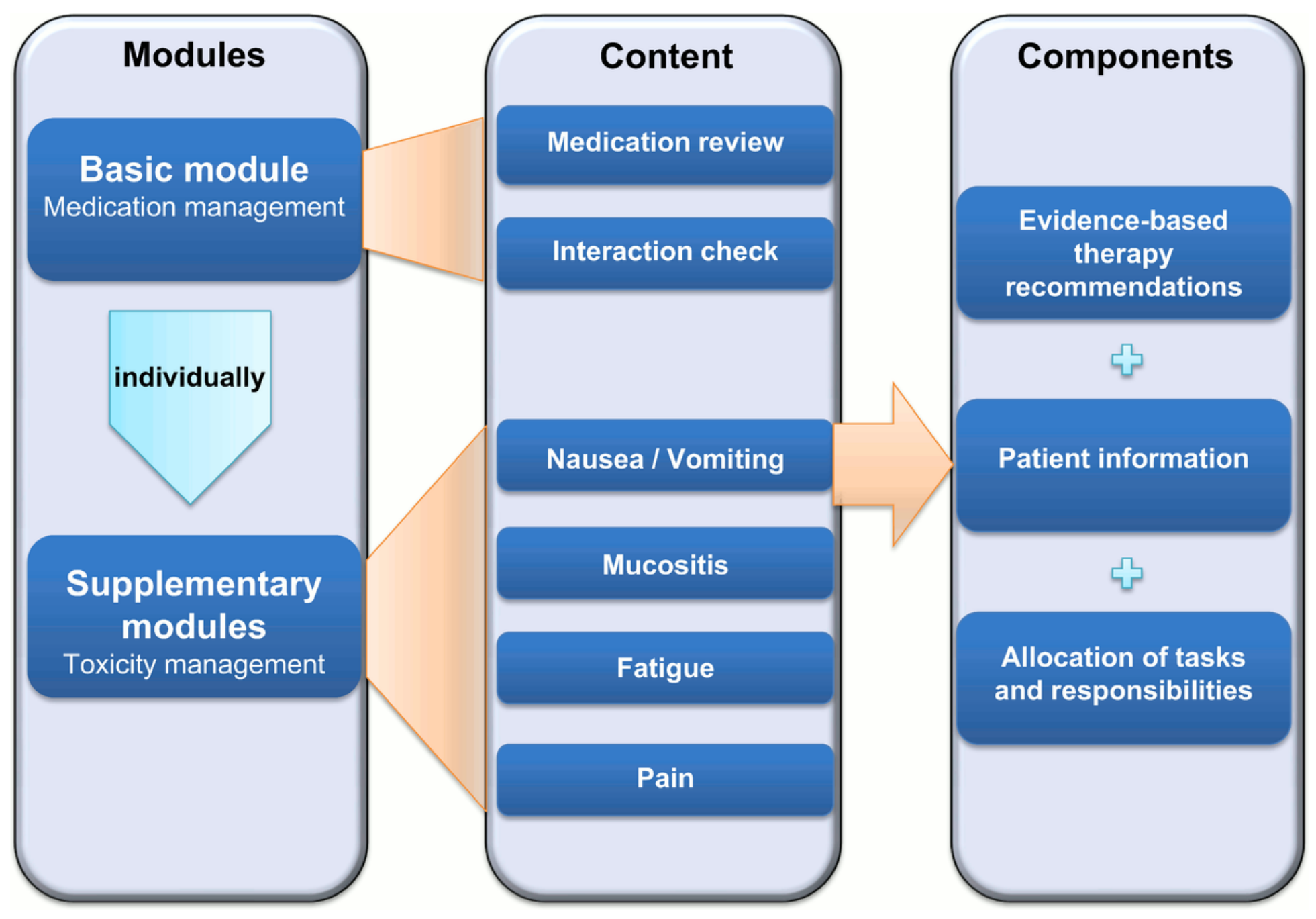

Figure 1

Structure and content of the MuMM intervention. 


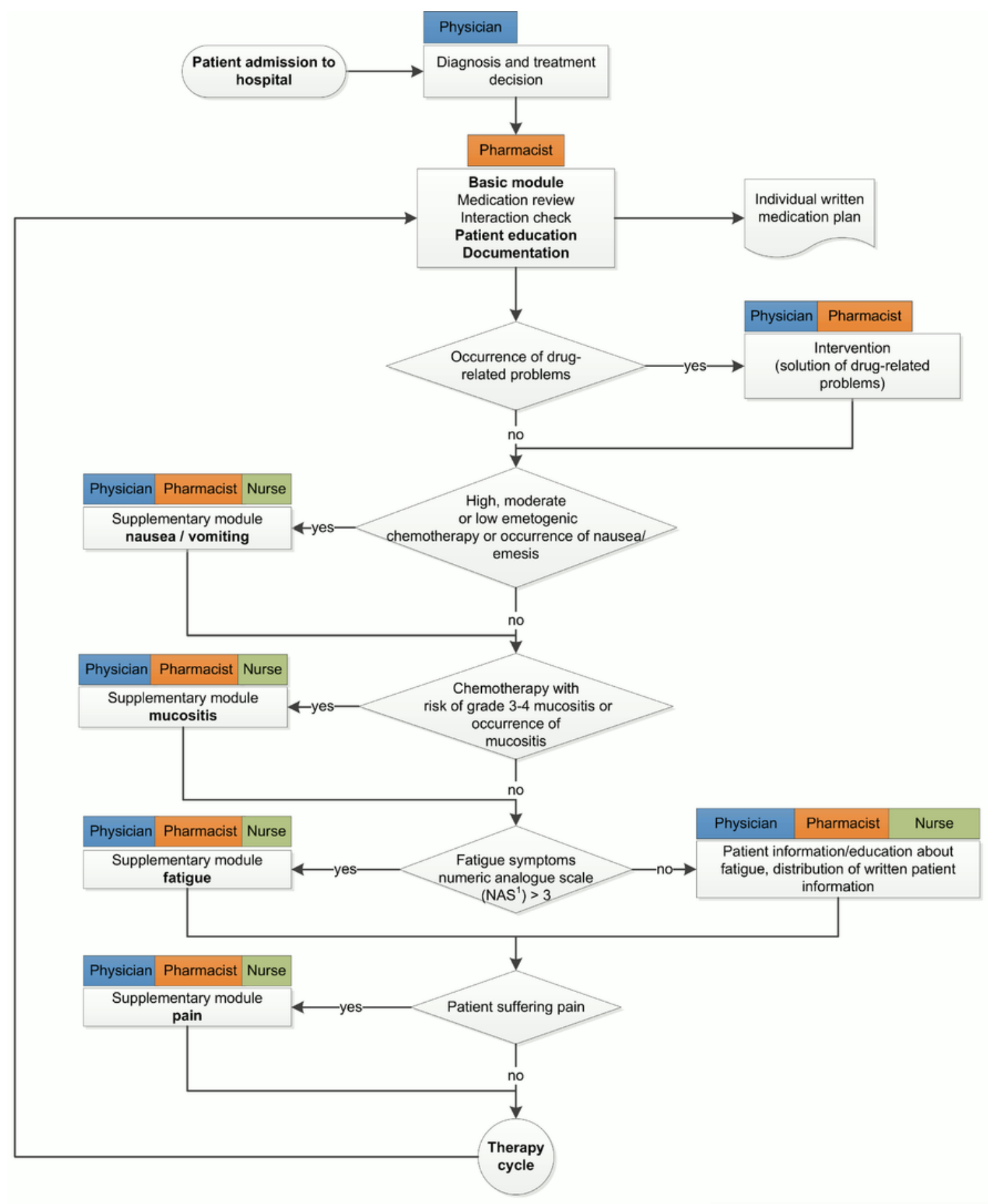

${ }^{1} \mathrm{NAS}=$ Numerical Analogue Scale

Explanation

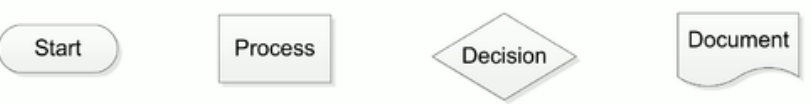

\section{Figure 2}

Algorithm of modular medication management in the intervention group. 


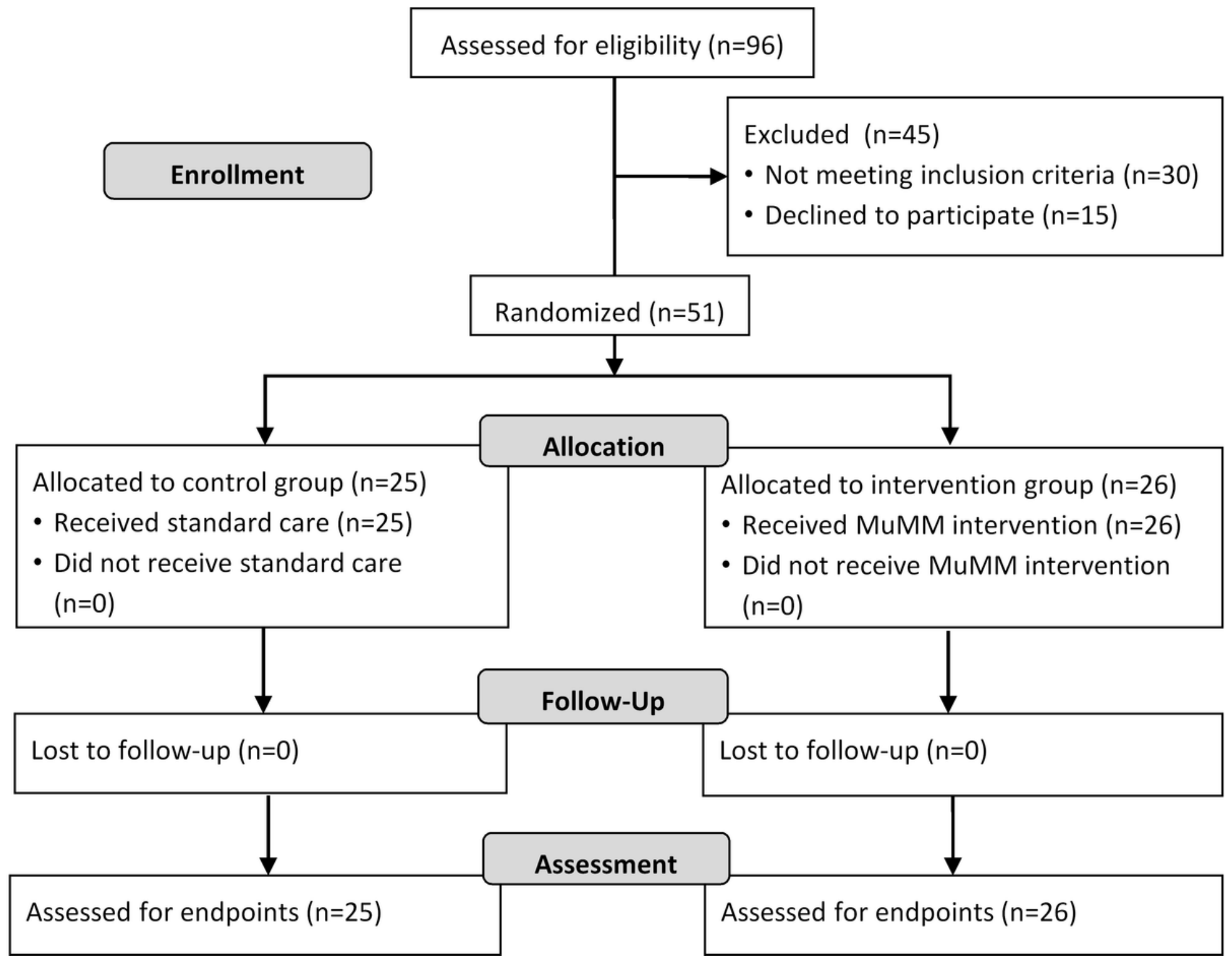

Figure 3

Patient recruitment flow diagram 

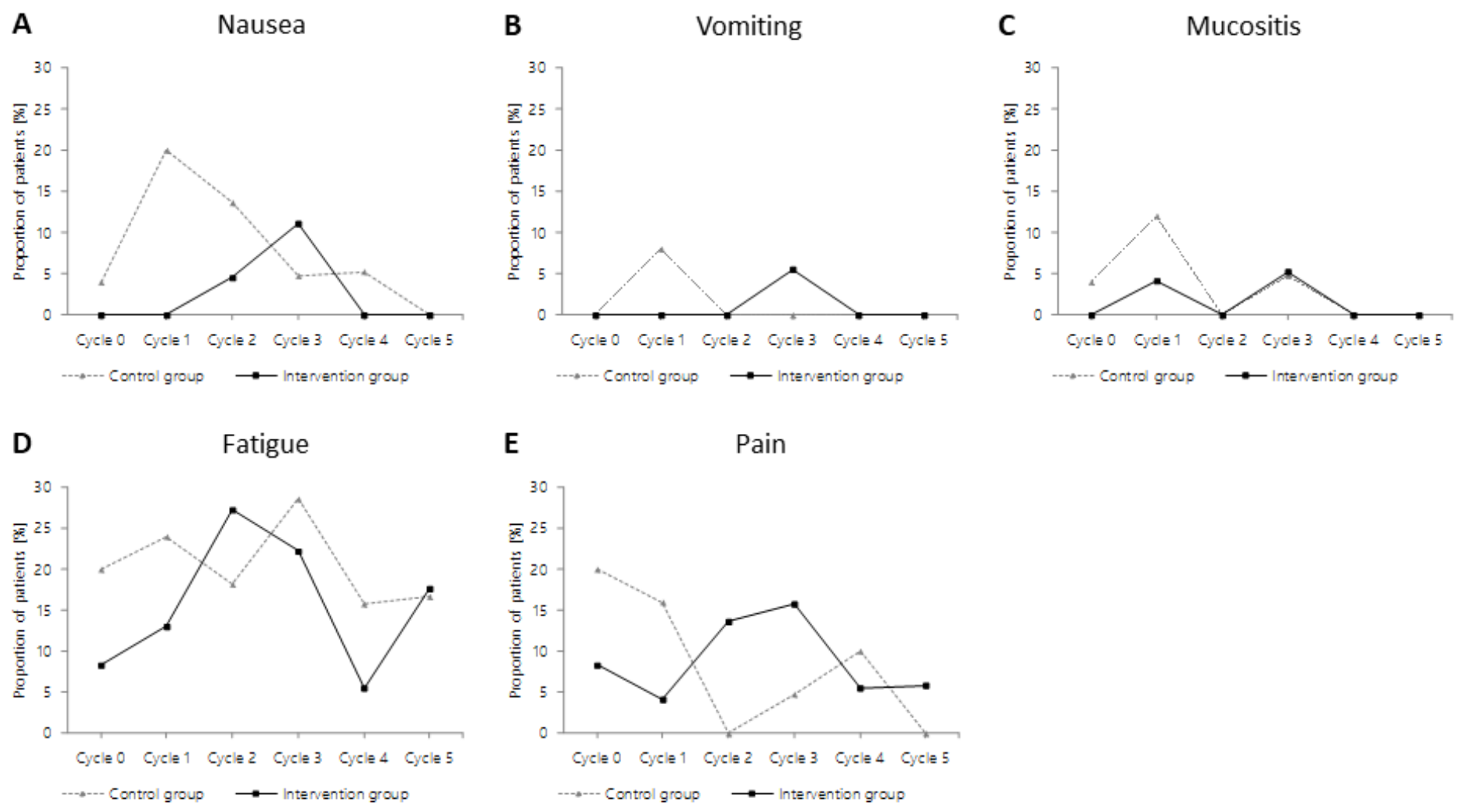

\section{Figure 4}

Proportion of patients per cycle and treatment group suffering from PRO-CTCAE score $\geq 75$ nausea (A), vomiting (B), mucositis (C), fatigue (D), or pain (E). 